\title{
A Service-Centric Architecture for Web Applications
}

\author{
Hans Albrecht Schmid \\ University of Applied Sciences Konstanz, Brauneggerstr. 55, D 78462 Konstanz \\ schmidha@fh-konstanz.de
}

\begin{abstract}
Service-centric architectures are gaining more and more importance, due to their benefits over conventional architectures. We propose a servicecentric Web application architecture that provides these benefits also for browser-based Web applications. An additional advantage is that B2C Web applications can be used also from B2B-clients. We extend OOHDM and its business process extension for service-centric Web applications architectures.
\end{abstract}

\section{Introduction and Related Work}

State-of-the-art Web applications are B2C (business-to-customer) applications where a customer may perform a spectrum of interactions like: navigation, the entering or updating of information, and the execution of business processes; with a business site or a similar site. Web application modeling and design methods (see e.g. [1,2]) mirror that spectrum, but do not reflect to a wide degree areas like business-to-business commerce (B2B) and Web services.

In B2B-commerce, the business processes of business partners communicate, without a person interacting. Web services, defining platform-independent and standardized interfaces for service invocation over the Web (compare [3,4]), are going to be predominantly used for B2B-communication [5]. Web service composition clients proposed for $\mathrm{B} 2 \mathrm{C}[6]$ are not browser-based.

B2B-aspects and Web services have so far not really been integrated into browserbased Web applications. Currently proposed is an architecture that provides composed Web services in parallel to the Web application [7]. E.g., Amazon allows external software applications to search and order articles by a parallel Web service interface [8]. [9] introduce Web services as additional data sources for Web applications in WebML.

In this paper, we propose to integrate Web services into Web applications so that we move from a data-centric or object-centric to a service-centric architecture. The objective of that architectural change is to obtain a system structure with well-defined services as interfaces, which are more stable, better suited for internal reuse of services and for system evolution, and also usable in a B2B-environment. The differences to [7] are that the services are not offered in parallel to the Web application, but directly integrated, and that we address problems related to business processes and conversations.

We present in section 2 the service-centric architecture for Web applications, propose a slight extension of OOHDM that allows to model it, and compare it with the state-of-the-art architecture. Section 3 shows how business processes fit into the architecture, and how conversational services are modeled. 


\section{Service-Centric Architecture of Web Applications}

The state-of-the-art Web application architecture (SAWAA), shown in Fig. 1. (left), is formed by: a browser; a Web front-end that is e.g. a servlet or a Java Server Page; a business object or application backend that is formed e.g. by persistent objects or Enterprise JavaBeans (EJB); and a database.
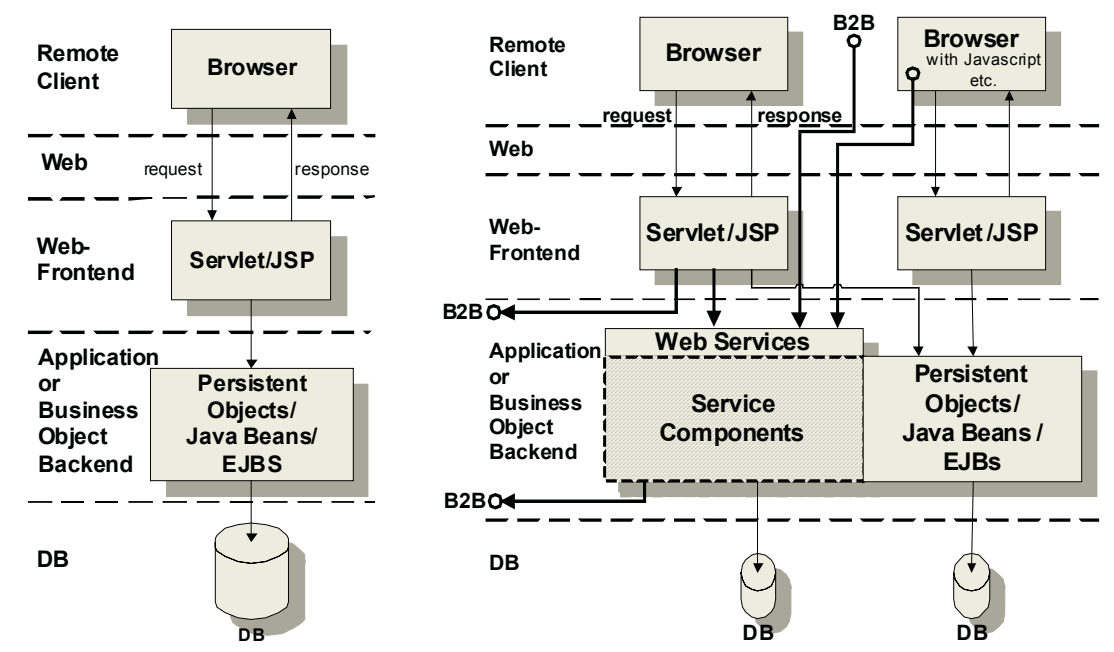

Fig. 1. State-of-the-art (left) and service-centric Web application architecture (right) with service and passive (top middle) and active (top right) HTML page

The interface of the Web front-end towards the browser defines a user request as an HTTP request type. This is a basic disadvantage of the conventional architecture, as the example of a hotel Web application shows. The hotel Web page contains information about the hotel, and a name and address input field and a button, which allow a prospective customer to request a hotel information flyer. The front-end servlet analyses the user input to the hotel page, detects a hotel information request, and processes that request together with the backend.

The problem is the Web front end receives a request for an action, like the hotelinfo request, that is defined implicitly by the Web page layout, like that of the hotel page (since the request contains the names and contents of the page input fields, buttons, etc.). If somebody modifies that page, e.g. by splitting the address in city and street address, the application logic code of the front-end must be modified. Further, another Web page of the same application cannot reuse the request. Moreover, no external B2B-application can make a hotel-info request to the Web application.

A service-centric architecture (SCA) (compare [6]) that consists of relatively fine-granular (possibly distributed) components with service interfaces may solve these problems. Therefore, we introduce a service-centric Web application architecture (SCWAA, see Fig. 1. right) which has a backend that provides businessrelated services via a service interface.

Consider the hotel-info example: a SCWAA provides a Web service called HotelService, which defines operations like sendHotelInfo( String customer, String ad- 
dress, Hotel theHotel) sending the hotel information to the customer. A service like HotelService is called an action service, since it provides independent actions like sendHotelInfo as operations. An action service is provided by a service component, which the invoker of the service does not (have to) know.

Operations of a business-related service may be invoked (see Fig. 1. right, thick arrowhead lines), typically without dynamic discovery and binding, either from the Web front-end, or from an active HTML page, or from an external application.

1. The front-end is strongly simplified if it invokes an action service, instead of performing the related responsibilities itself. For example, if the hotel-info servlet invokes the sendHotelInfo-operation of the HotelService on a user hotel information request, its only responsibility is to return the next page as response.

2. An HTML page may invoke directly a Web service in a function call of a scripting language, like JavaScript, e.g. with Web Service Behavior libraries from Microsoft. We use that feature to invoke an action service, like sendHoteInfo (see Fig. 1., top right) directly from the page, like the hotel page, from which a user triggers the execution of an action. As a consequence, we achieve a clear separation of concerns: the HTML page with the scripting code has the responsibility for data entry and for the invocation of the action; and the front-end has the responsibility for dynamic navigation. Therefore, we prefer this architectural option.

3. An external B2B-application may invoke an action service provided by a Web application if it has the access rights. Thus, B2B-interactions may be executed with a browser-driven Web application. For example, a tourist information Web application can request a hotel flyer with a B2B-communication from the Web application of the selected hotel, invoking the provided HotelService.

Further, a Web application may invoke externally provided services as additional data sources from the Web front-end or from a backend service component, either public services like train timetable information, or B2B-services from another Web application.

\section{Service Representation by OOHDM}

We propose to represent an action service, like the HotelService, in the OOHDM conceptual schema as an interface with a stereotype like <<action service interface $>>$, which refines the stereotype $<<$ service interface $>>$ (see Fig. 2. left).

When a Web application provides and implements a Web service, the OOHDM conceptual schema may represent not only the Web service interface, but also the component implementing it, like the HotelServiceComponent that implements the service interface and collaborates with persistent classes like Hotel and Customer (see Fig. 2. left). When a Web application invokes an external service, it is not concerned about the implementation of the service. Therefore, the conceptual schema represents only the service interface, and does not show how it is implemented.

The OOHDM navigational schema (see Fig. 2. right) models the responsibilities of the front-end by nodes, like HotelInfoNode, that may have action methods, like requestHotelInfo. If a method of a node invokes a Web service, the node has a directed association to the service interface, which is represented by a circle.

Summarizing, a SCWAA provides the business-related services as a well-defined, clear and stable interface to the backend components, which might, as an additional advantage, also be realized by an existing application system in the context of enter- 

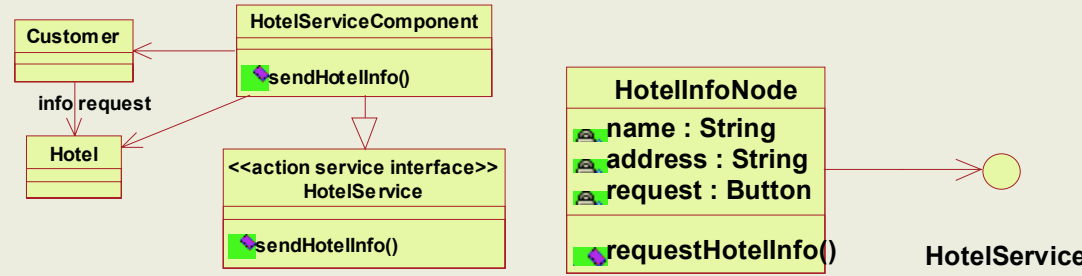

Fig. 2. OOHDM conceptual schema (left) with HotelService provided by HotelServiceComponent, and OOHDM navigational schema (right) with a directed association from the HotelInfoNode to the HotelService

prise application integration (EAI). Moreover, also external B2B-applications may invoke services that are primarily provided for internal use.

\section{Realizing Business Processes with Web Services}

A B2C business process [2], like the check-out process of a Web shop, guides a user in a prescribed order through several activities, like Login, ConfirmItems, SelectShippingAddress, etc. During each activity, the user enters specific data like the userlogin data; possibly after the activity has shown data from a database as default entries, like a previously entered shipping address.

A Web application with a SCWAA provides a business process service, like a CheckOutService (see Fig. 3.). The difference to an action service is that a business process service includes a set of interrelated operations, often one per activity of the business process. For example, CheckOutService provides the operations enterLoginData( $u$ : UserData), enterOrderedItems(...), etc. The operation confirmOrder collects all entered data and creates a valid order. All operations or a subset of them must be invoked in a particular order, called the conversation protocol, to complete the business process. Thus, a business process service defines a conversation, which has a state, in contrast to a sequence of stateless invocations. Both the definition of a "conversational" service, and the interactions between such a service and a client like the Web front-end are more complex than for single, independent invocations.

The operations of a business process service may be invoked, same as those of an action service, from an active HTML page, from the front-end (we use the second option for the reasons described in section 2), or from a B2B-application.

We model a SCWAA for business processes with OOHDM by including the business process service interface into the OOHDM conceptual schema and the invocation of its operations in the OOHDM navigational schema.

We use the check-out process of the Web shop example and present it with a SCWAA, to allow a comparison with the SAWAA shown in [2]. The conceptual schema (see Fig. 3. left) includes the business process service CheckOutService implemented by the component CheckOut. A business process like CheckOutProcess is represented by a process class with the stereotype $<<$ root activity $>>$ with child activities as attributes. It has as responsibilities the sequencing among the activities, and providing the pages to be displayed by an activity. A process is light-weight in comparison to the SAWAA, since the process service takes over all responsibilities related to the handling of process-related data entered by the user. 

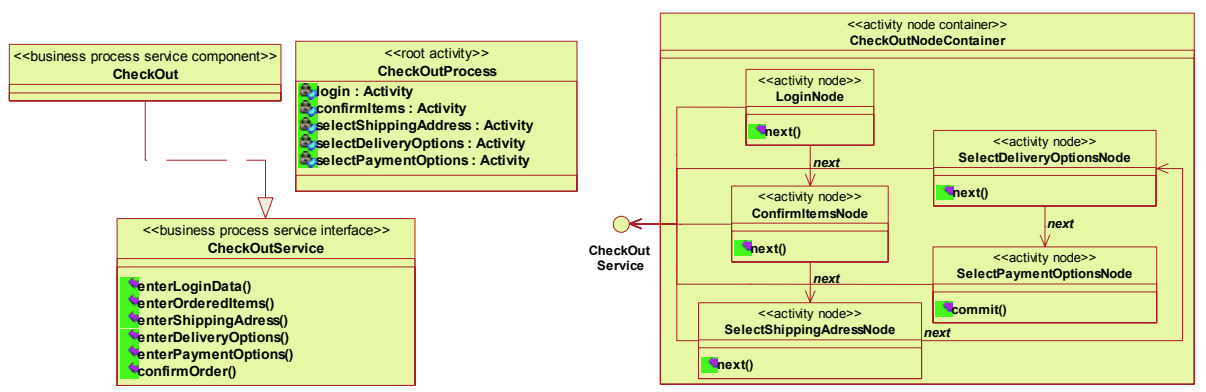

Fig. 3. OOHDM conceptual schema (left) and navigational schema (right) of the check-out process of a CD Webshop
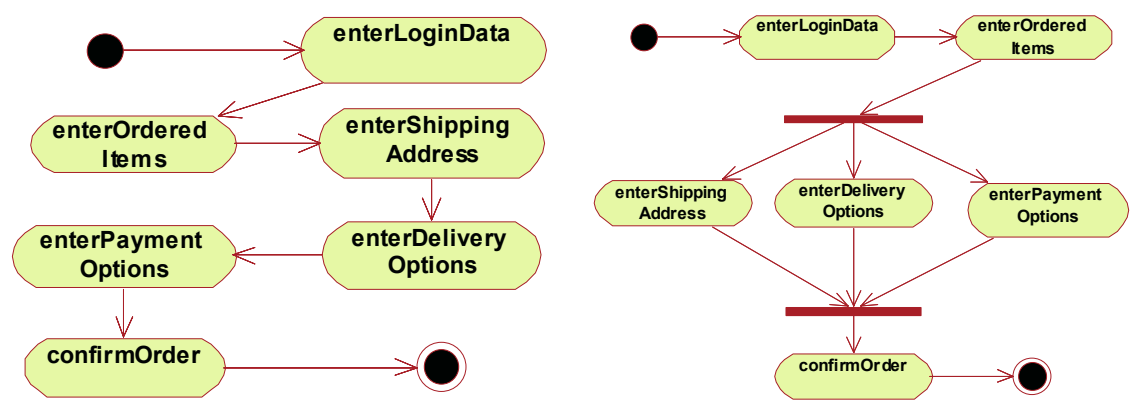

Fig. 4. Activity diagrams for CheckOutService conversation protocol specification

To specify the conversation protocol, we add for each business process service interface an UML activity diagram that represents the conversation protocol (see Fig. 4. for two alternative conversation protocols), to the conceptual schema. This is consistent with the OOHDM business process specification [2] that uses an activity diagram to define the correct sequences of activity execution.

The navigational schema (see Fig. 3. right) is similar as in the SAWAA, except for the activity nodes with methods invoking services. The differences is that the activity nodes have a reference to a process service, like CheckoutService, since their methods invoke an operation of it.

The navigational schema, implemented by the front-end, is designed so that it guides a user through the activities of a business process and presents the Web pages in an order that complies to the protocol specification.

\section{Conclusions}

We have proposed a service-centric architecture for Web applications that transfers the benefits of service-centric architectures to Web applications. It has been successfully used to implement several Web applications with business processes.

Due to lack of space, we could not address light-weight solutions for problems related to service-centric business process execution, like conversation protocol specification, protocol compliance verification and conversation routing. The light-weight 
solutions are adapted to the Web application scenarios which are simpler and less general than those for cooperating business process in the Web service community (see [10]).

\section{Acknowledgements}

My thanks are due to Marco Pfeifer and Thorsten Schneider for his help with the figures; and to the Ministerium fuer Wissenschaft und Forschung, BadenWuirttemberg for a partial support of the project.

\section{References}

1. D. Schwabe, G. Rossi: "An object-oriented approach to web-based application design". Theory and Practice of Object Systems (TAPOS), V.4\#4, pp.207-225, October, 1998

2. H. A. Schmid, G. Rossi “ Modeling and Designing Processes in E-Commerce Applications“. IEEE Internet Computing, Jan./Feb. 2004, pp.2-10

3. F.Leymann, D.Roller, M.T.Schmidt: Web Services and Business Process Management; IBM Systems Journal Vol.41, No2, 2002

4. W3C: Web Services Architecture Requirements. see: http://www.w3.org/TR/wsa-reqs

5. T.Andrews et al: Specification of the Business Process Execution Language for Web Services Version 1.1; at: http://ifr.sap.com/bpel4ws/

6. Procs. IEEE International Conference on Services Computing, Shanghai, China, 2004, IEEE Press, Los Alamitos, 2004

7. I.Manolescu, S.Ceri, M.Brambilla, P.Fraternali, S.Comai: "Exploring the combined potential of Web sites and Web services". Poster, Procs World Wide Web 2003

8. Amazon Inc.: Amazon Web Services; http://www.amazon.com/Webservices

9. M.Brambilla, S.Ceri, S.Comai, P.Fraternali, I.Manolescu: "Model-driven Specification of Web Services Composition and Integration with Data-Intensive Web Applications".

10. G.Alonso, F.Casati, H.Kuno, V.Machiraju: Web Services - Concepts, Architectures and Applications; Springer, Berlin, 2004 